\title{
Molecular and Sugar-Binding Heterogeneity of C-Type Lectins from Osmerus (Spirinchus) lanceolatus Eggs
}

\author{
Shigeki Sugawara, Masahiro Hosono, Yukiko Ogawa, Motoaki TakaYanagi, and Kazuo Nitta* \\ Cancer Research Institute, Tohoku Pharmaceutical University; 4-4-1 Komatsushima, Aoba-ku, Sendai 981-8558, Japan. \\ Received November 4, 2004; accepted March 5, 2005
}

Two C-type lectins (OLLafs and OLLafl) were isolated from Osmerus (Spirinchus) lanceolatus eggs using asialofetuin-Sepharose column. OLLafs and OLLafl were eluted with $0.2 \mathrm{M}$ sucrose and $0.2 \mathrm{M}$ lactose from the same column, respectively. OLLafl has been estimated to be a heterodimeric protein composed of $\mathrm{H}$ - and $\mathrm{L}$-subunit and involved C-type lectin like domain (CTLD). In this study we revealed that OLLafs was a homodimeric protein composed of L-subunit of OLLafl. Although adding EDTA diminished the hemagglutinating activity of OLLafs, the activity of OLLafl was not influenced. Recombinant lectins (rOLLafl-H and -L) and mutant lectins

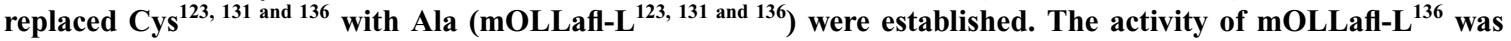
comparable to rOLLafl-L, and rOLLafl-H was 15 times lower than rOLLafl-L. On the other hand, the activity of mOLLafl-L ${ }^{123}$ and mOLLafl-L ${ }^{131}$ were lower than that of rOLLafl-H. Therefore, Cys ${ }^{136}$ may not participate in hemagglutinating activity of rOLLafl-L. In contrast, $\mathrm{Cys}^{123}$ and $\mathrm{Cys}^{131}$ may partially contribute this activity. Although hemagglutination inhibition profiles of rOLLafl-L, rOLLafl-H and mOLLafl-L ${ }^{136}$ were similar, mOLLafl- $\mathrm{L}^{131}$-induced hemagglutination was not inhibited by any sugars tested even at a concentration of $150 \mathrm{mM}$. Then, Cys ${ }^{131}$ may directly contribute to the sugar-binding capacity of OLLafl. Affinities of mOLLafl-L ${ }^{123}$ for these sugars were lower than the others. These results suggest that $\mathbf{C y s}^{136}$ might contribute to the intermolecular disulfide bond in the rOLLafl-L dimer, and that the intramolecular disulfide bond concerning Cys ${ }^{131}$ might important for lectin activity.

Key words C-type lectin; fish egg; subunit composition; heterogeneity

Animal lectins are originally characterized by their carbohydrate-binding or -recognition activity, and their own structural motifs, e.g. M-, L-, P-, I-, and R-type lectins and calnexin in addition to the classical families of C-type and galectins. ") "C-type" family was derived from $\mathrm{Ca}^{2+}$-dependent lectins involving consensus motif of carbohydrate recognition domain (CRD), which is well established to concern with the binding form to their hapten sugar or $\mathrm{Ca}^{2+}$ ion. ${ }^{2)}$ Recently, the notion of C-type CRD was reconsidered to be a C-type lectin-like domain (CTLD) by focusing the "fold" of the modules regardless carbohydrate-binding capacity. ${ }^{1)}$ In fish, several C-type lectins were isolated from eggs, ${ }^{3)}$ serum ${ }^{4)}$ and skin mucus, ${ }^{5)}$ and predicted from cDNA library or database. ${ }^{6-11)}$ Most of fish C-type lectins found so far are classified into group VII composed of "free CTLD" bearing "EPN" or "QPD" motifs. Among these, Anguilla japonica lectin-2 (AJL-2) from skin mucus of Japanese eel possesses unusual aspects in comparison with other lectins as follows: (1) AJL-2 does not bind to mannose but to lactose nevertheless it has "EPN" motif, and (2) AJL-2 does not require $\mathrm{Ca}^{2+}$ ion for its hemagglutinating activity. Lectins found in skin mucus are thought to participate in the host defense. ${ }^{5)}$ More recently, it was reported that GCOL (C-type lectin isolated from gibel carp eggs) was associated and translocated with cortical granules during oocyte maturation and egg fertilization. ${ }^{3)}$ In addition, GCOL is phosphorylated and dephosphorylated during fertilization and early embryo development, indicating that GCOL may participate in polyspermy blocking and in embryonic development protection.

In the previous study, we reported about C-type lectin isolated from Osmerus (Spirinchus) lanceolatus eggs (OLLafl). OLLafl was eluted from asialofetuin-Sepharose column with 0.2 м lactose (Hosono M., Sugawara S., Ogawa Y., Khono T.,
Takayanagi M., and Nitta K., unpublished observation). According to the results of sodium dodecylsulfate-polyacrylamide gel electrophoresis (SDS-PAGE) and cDNA sequence analysis, OLLafl was composed of two almost identical $(95 \%)$ subunits, $16 \mathrm{kDa}$ of $\mathrm{H}$-subunit and $15 \mathrm{kDa}$ of L-subunit (OLLafl-H and -L), involving "EPN" motif of CTLD. OLLafl recognized lactose and D-galactose, but not D-mannose, in a $\mathrm{Ca}^{2+}$-independent manner. $\mathrm{H}$ - and L-subunit of OLLafl were separately expressed in Escherichia coli, and hemagglutinating activities of recombinant subunits were higher than that of OLLafl. Here we describe about molecular heterogeneity of native OLLafl purified by asialofetuinSepharose with lactose, and related but distinct C-type lectin, which was eluted from the same column with $0.2 \mathrm{M}$ sucrose (OLLafs) separately. In this study, we elucidated that C-type lectins having multiple subunit composition and carbohydrate-binding specificity were included in $O$. lanceolatus eggs.

\section{MATERIALS AND METHODS}

Materials Shishamo smelts (O. lanceolatus) were collected in the sea near Hokkaido, Japan. Human erythrocytes were kindly provided by the Miyagi Prefecture Red Cross Transfusion Center, and rabbit erythrocytes were purchased from Nippon Biotest Laboratories (Tokyo, Japan). Fetuin was from Sigma (St. Louis, MO, U.S.A.). Cyanogen bromideactivated Sepharose 4B was from Amersham Pharmacia Biotech (Uppsala, Sweden). DEAE-Toyopearl anion exchange resin was from Toso (Tokyo, Japan). Ni-NTA HisBind Resin was from Novagen (Darmstadt, Germany). Other reagents were of the highest analytical grade.

Preparation of Affinity Resin Asialofetuin was obtained by mild acid hydrolysis of fetuin. ${ }^{12)}$ Asialofetuin- 
Sepharose was prepared using CNBr-activated Sepharose 4B as described by March et al. ${ }^{13)}$

Preparation of two OLLs Saline extracted fraction obtained from acetone-dried powder of $O$. lanceolatus eggs was separated on a DEAE-Toyopearl column as described previously. ${ }^{14)}$ Stepwise gradient of phosphate buffer (PB) (pH 6.8) from $10 \mathrm{~mm}$ to $400 \mathrm{~mm}$ was done and each fraction was collected. D30 (100 mg) eluted with $30 \mathrm{~mm}$ PB was applied to an asialofetuin-Sepharose column $(1.0 \times 15 \mathrm{~cm})$ equilibrated with $10 \mathrm{~mm} \mathrm{~PB}$ ( $\mathrm{pH} 7.2$ ) containing $0.15 \mathrm{~m} \mathrm{NaCl}$ (PBS). The adsorbed fraction was eluted with PBS containing $0.2 \mathrm{M}$ lactose (OLLafl). Separately, we attempted to elute non-specifically adsorbed protein by $0.2 \mathrm{M}$ sucrose (OLLafs) prior to lactose. Each adsorbed fraction was dialyzed against distilled water for $3 \mathrm{~d}$, and then lyophilized.

Electrophoresis and Protein Assay SDS-PAGE and native-PAGE were performed as described by Laemmli ${ }^{15)}$ and Davis, ${ }^{16)}$ respectively. After electrophoresis, the gel was stained with Coomassie Brilliant Blue (CBB). Protein concentration was determined by the method of Lowry et al. ${ }^{17)}$ using DC protein assay kit (Bio-Rad, Hercules, CA, U.S.A.) and bovine serum albumin (BSA) as a standard.

Following SDS- or native-PAGE as described above, separated proteins were transferred to a polyvinylidene difluoride (PVDF) membrane in transfer buffer $(25 \mathrm{~mm}$ Tris, $192 \mathrm{~mm}$ glycine) at $60 \mathrm{~V}$ for $3 \mathrm{~h}$. Blotted membrane was stained with $\mathrm{CBB}$ and the protein bands were cut, reduced with dithiothreitol (DTT), and pyridylethylated. The membrane strips were subjected to an Applied Biosystems 494 gas-phase sequencer (Applied Biosystems, Foster City, CA, U.S.A.) and sequenced by automated Edman degradation.

Hemagglutination and Inhibition Assays Hemagglutination assay was performed using $2 \%$ rabbit erythrocyte suspension in PBS in the presence or absence of EDTA (5 mM) as described previously. ${ }^{18)}$ The hemagglutination inhibition assay was performed by incubating $25 \mu$ l aliquots of lectin ( 8 times higher than the minimum concentration of protein required for positive agglutination) and various concentrations of mono- or oligosaccharides in PBS with the same volume of erythrocyte suspension.

Site-Directed Mutagenesis cDNAs of $\mathrm{H}$ - and L-subunit of OLLafl had been constructed with pGEM vector and cloned into the expression vector pET16b (Novagen) (data not shown). Both subunits of OLLafl included seven Cys residues. To elucidate importance of Cys residues distributed especially in the neighbor of C-terminal CTLD region (Fig. $3 \mathrm{~B})$, we produced three mutant cDNA constructs containing single mutation to substitute Cys to Ala of OLLafl-L using Gene Tailor site-directed mutagenesis system (Invitrogen, San Diego, CA, U.S.A.) according to the standard manufacturer's protocol. pGEM inserted L-subunit cDNA was methylated with DNA methylase included in the kit before mutation. To make three mutants of OLLafl-L, the primer set of 5'-GATGGAACGATTCTCCAGCCGACCAGGAAC-3' and 5' - TGGAGAATCGTTCCATCGGTGATCAGC-3' for mOLLafl-L ${ }^{123}, 5^{\prime}$-AGGAACTCGGCTTCATCGCCTCCTTCAAAC- $3^{\prime}$ and $5^{\prime}$-GATGAAGCCGAGTTCCTGGTCACATGG-3' for mOLLafl-L ${ }^{131}$, and 5'-TCTGCTCCTTCAAACTCGCCTAACATATGG-3' and 5'-GAGTTTGAAGGAGCAGATGAAGCCGAG-3' for mOLLafl-L ${ }^{136}$ (substituted nucleotides were noted by italic letter) were used. Point muta- tion was introduced by polymerase chain reaction (PCR) method using Pyrobest (Takara Bio Inc., Ohtsu, Japan) as a DNA polymerase following the step of $30 \mathrm{~s}$ at $94{ }^{\circ} \mathrm{C}, 30 \mathrm{~s}$ at $55^{\circ} \mathrm{C}$, and $3.5 \mathrm{~min}$ at $72^{\circ} \mathrm{C}$ for 20 cycles. PCR products were transformed into DH5 $\alpha$ cells. Then, the positive clone was isolated and the sequence was confirmed using Dye terminator cycle sequencing kit and CEQ8000 capillary sequencer (Beckman Coulter, Fullerton, CA, U.S.A.). cDNA fragments bearing single point mutation were ligated to $\mathrm{pET} 16 \mathrm{~b}$ vector at Nde I and Bam HI sites, and then transformed into E. coli BL21(DE3)pLysS host cells grown in Luria-Bertani (LB) medium containing $50 \mu \mathrm{g} / \mathrm{ml}$ carbenicillin and $34 \mu \mathrm{g} / \mathrm{ml}$ chloramphenicol.

Expression and Purification of Recombinant and Mutant OLLs Recombinant OLLafl-H and -L (rOLLafl-H and -L) and three mutants (mOLLafl- $\mathrm{L}^{123}$, mOLLafl-L ${ }^{131}$ and mOLLafl-L ${ }^{136}$ ) were expressed in the BL21(DE3)pLysS transformant cells inducing with $1 \mathrm{~mm}$ IPTG for $6 \mathrm{~h}$ at $37^{\circ} \mathrm{C}$. IPTG-induced $E$. coli cells were harvested by centrifugation at $6000 \times \boldsymbol{g}$ for $15 \mathrm{~min}$, and resuspended with sonication buffer ( $50 \mathrm{~mm}$ Tris- $\mathrm{HCl}$ containing $50 \mathrm{~mm} \mathrm{NaCl}, \mathrm{pH} 8.0$ ) and stored at $-80^{\circ} \mathrm{C}$. To disrupt cell wall, frozen cells were thawed at $37^{\circ} \mathrm{C}$ and sonicated. The cell extract was centrifuged at $20000 \times \boldsymbol{g}$ for $20 \mathrm{~min}$, and the resulting inclusion body was collected and washed with sonication buffer containing 4\% Triton X-100 followed by $\mathrm{H}_{2} \mathrm{O}$.

The inclusion body derived from culture medium $(200 \mathrm{ml})$ was dissolved in $100 \mathrm{~mm} \mathrm{~PB}(\mathrm{pH} 8.0)$ containing $6 \mathrm{~m}$ guanidine- $\mathrm{HCl}(0.5 \mathrm{ml})$, and subjected to Ni-NTA His-Bind Resin (Qiagen, Hilden, Germany) column equilibrated with starting buffer (100 mм PB containing $8 \mathrm{~m}$ urea, $\mathrm{pH} 6.0)$. After washing with starting buffer ( 20 bed volumes), the recombinant proteins were eluted with $20-250 \mathrm{~mm}$ imidazole.

Refolding of Recombinant and Mutant OLLs The recombinant proteins were refolded following the procedure of Ewart et al. ${ }^{19)}$ with modifications. Briefly, the eluate was diluted with $20 \mathrm{~mm}$ PB ( $\mathrm{pH} 8.0$ ) containing $2 \mathrm{~mm}$ 2-mercaptoethanol $(\mathrm{ME})$ and $8 \mathrm{M}$ urea until the final protein concentration was up to $100 \mu \mathrm{g} / \mathrm{ml}$. The solution was dialyzed against $50 \mathrm{~mm}$ Tris- $\mathrm{HCl}$ (pH 8.0) containing $100 \mathrm{~mm} \mathrm{NaCl}$ and $1 \mathrm{~mm}$ $\mathrm{CaCl}_{2}$ at $4{ }^{\circ} \mathrm{C}$ for $48 \mathrm{~h}$, and subsequently against $50 \mathrm{~mm}$ $\mathrm{NH}_{4} \mathrm{HCO}_{3}$ at $4{ }^{\circ} \mathrm{C}$ for $72 \mathrm{~h}$. The resulting solution was evaporated in vacuo, and redissolved in PBS.

\section{RESULTS}

Purification and Hemagglutinating Activity of OLLafs and OLLafl As shown in Fig. 1A, D10 fraction eluted with $10 \mathrm{~mm}$ PB contained the rhamnose-binding lectin (OLL). Fig. $1 \mathrm{~B}$ shows a chromatogram of saline extract from $O$. lanceolatus eggs on a DEAE-Toyopearl column. Since D30 eluted with $30 \mathrm{~mm}$ PB contained distinct hemagglutinating activity from D10, affinity purification was performed using asialofetuin-Sepharose column. OLLafl was eluted with $0.2 \mathrm{~m}$ lactose from the same affinity column described above. To washout non-specific adsorbent, $0.2 \mathrm{~m}$ sucrose was used before lactose elution. Unfortunately, almost of all adsorbent was eluted with $0.2 \mathrm{M}$ sucrose and only trace amount of proteins were obtained in the latter elution step (Fig. 1C). The sucrose-eluted fraction, OLLafs, showed slightly higher hemagglutinating activity than OLLafl for rabbit erythrocytes (Table 1). The 
A
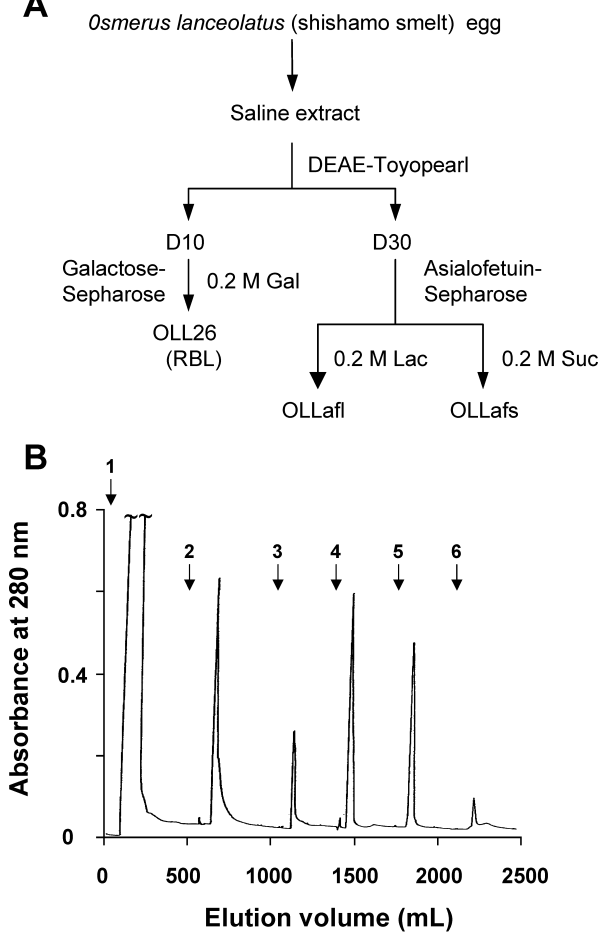

C

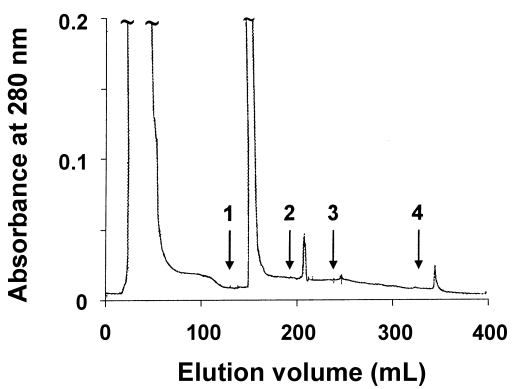

Fig. 1. Purification of OLLafs

OLLafs was purified by DEAE-Toyopearlion exchange and asialofetuin-Sepharose affinity column chromatographies (A). RBL, rhamnose-binding lectin. Lectin activities were obtained in D10 and D30 eluted with $10 \mathrm{~mm}$ and $30 \mathrm{~mm}$ phosphate buffer (PB) (pH 6.8), respectively, from DEAE-Toyopearl column (B). Numbers from 1 to 6 with arrows indicate a stepwise gradient of PB, 10,30,50,100, 200, and $400 \mathrm{~mm}$, respectively. D30 fraction $(100 \mathrm{mg})$ was dissolved in $10 \mathrm{~mm}$ phosphate buffer $(\mathrm{pH} 7.2)$ containing $0.15 \mathrm{M} \mathrm{NaCl}$ and applied to the asialofetuin-Sepharose column $(\mathrm{C})$. After washing with the same buffer, the column was eluted successively with (1) $0.2 \mathrm{M}$ sucrose, (2) $0.02 \mathrm{M}$ lactose, (3) $0.2 \mathrm{M}$ lactose and (4) $0.1 \mathrm{M}$ acetate buffer (pH 4.0) containing $0.5 \mathrm{M}$ $\mathrm{NaCl}$ as indicated by arrows.

Table 1. Hemagglutinating Activity of OLLafs, OLLafl and Recombinant Lectins

\begin{tabular}{lcc}
\hline \hline & \multicolumn{2}{c}{ Hemagglutinating activity $^{a)}$} \\
\cline { 2 - 3 } Lectin $^{b)}$ & -EDTA & + EDTA \\
\hline afs & 20 & $>360$ \\
afl & 50 & 50 \\
rafl-H & 3.1 & $>25$ \\
rafl-L & 0.2 & $>11$ \\
mafl-L ${ }^{123}$ & 10 & $>80$ \\
mafl-L & 15 & 60 \\
mafl-L & & $>18$
\end{tabular}

a) Hemagglutinating activity was defined as minimum amount of lectin concentration $(\mu \mathrm{g} / \mathrm{ml})$ required for positive agglutination of rabbit erythrocytes with or without 5 mм EDTA. b) Lectins abbreviated are; afs, OLLafs; afl, OLLafl; rafl-H and -L, recombinant OLLafl-H and -L, respectively; mafl- $\mathrm{L}^{123}$, $-\mathrm{L}^{131}$ and $-\mathrm{L}^{136}$, mutant lectins replaced $\mathrm{Cys}^{123}, \mathrm{Cys}^{131}$ and $\mathrm{Cys}^{136}$ to Ala, respectively.
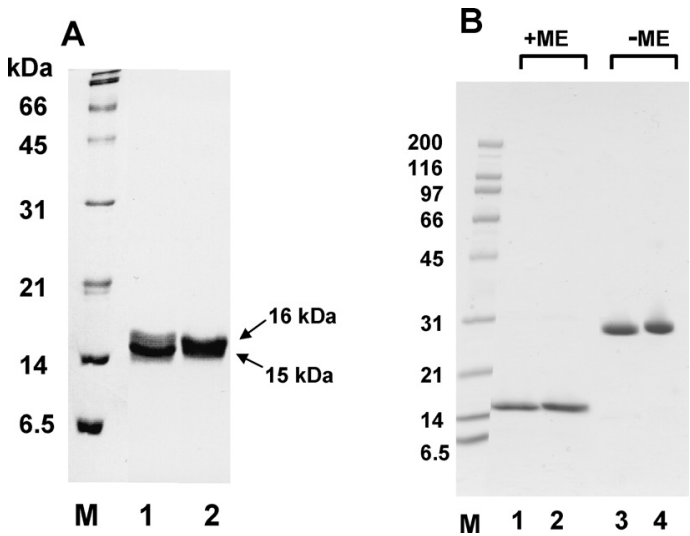

Fig. 2. SDS-PAGE Pattern of OLLafs and OLLafl

SDS-polyacrylamide gel electrophoreses were performed using homogeneous $20 \%$ gel (A) and 10-20\% gradient gel (B). OLLafs (lanes 1 and 3), and OLLafl (lanes 2 and 4) were treated with (lanes 1 and 2) or without (lanes 3 and 4) 2-mercaptoethanol (ME). M, marker proteins.

addition of $5 \mathrm{~mm}$ EDTA resulted in a lowering of the hemagglutinating activity of OLLafs but not of OLLafl indicating that OLLafs may be a $\mathrm{Ca}^{2+}$-dependent lectin (Table 1).

SDS-PAGE Pattern of OLLafs and OLLafl OLLafs showed one main band corresponding to molecular mass of $15 \mathrm{kDa}$ with overlaid faint band of $16 \mathrm{kDa}$. On the contrary, OLLafl was composed of two bands of $16 \mathrm{kDa}$ (H-subunit) and $15 \mathrm{kDa}$ (L-subunit) with even density on SDS-PAGE using a homogeneous $20 \%$ separation gel under reducing conditions (Fig. 2A). Therefore, we estimated that OLLafl was heterodimeric $(\mathrm{H} / \mathrm{L})$ protein. Using $10-20 \%$ gradient gel, OLLafs and OLLafl showed a single band of $15 \mathrm{kDa}$ under reducing conditions, and a slightly broad single band of $c a$. $30 \mathrm{kDa}$ under non-reducing conditions (Fig. 2B). The discrepancy of electrophoresis pattern under reducing conditions shown in Figs. 2A and B may result from the type of separation gel, homogeneous or gradient. The main band and the overlaid faint band of OLLafs shown in Fig. 2A were separated and sequenced. Both bands showed the N-terminal sequence of L-subunit (Fig. 3B), suggesting that OLLafs was a homodimer (L/L) (data not shown).

Sugar Specificity of OLLafs and OLLafl OLLafs-induced hemagglutination was inhibited by $N$-acetyl-D-glucosamine (D-GlcNAc), melibiose, D-glucose, lactose, Dgalactose, D-mannose and sucrose, but not by L-rhamnose, $\mathrm{N}$ acetyl-D-galactosamine (D-GalNAc) and lactulose (Table 2). Minimum concentrations of methyl- $\alpha$-D- and methyl- $\beta$-Dglucoside causing obvious inhibition were $3.75 \mathrm{~mm}$ and $1.89 \mathrm{~mm}$, respectively (data not shown). On the other hand, the effective inhibitors for OLLafl-induced hemagglutination were L-rhamnose, D-galactose, lactulose, melibiose and lactose, but not D-glucose, D-mannose, D-GalNAc, D-GlcNAc and sucrose. These results indicate that OLLafs and OLLafl have affinity for glucosyl residue and galactosyl residue, respectively. Since OLLafs is estimated to be a dimer of OLLafl-L $(\mathrm{L} / \mathrm{L})$ as described above, it belongs to a member of group VII C-type lectin. It must be noted that OLLafs has characteristic carbohydrate-binding property, because group VII C-type lectins commonly have affinity for D-mannose or D-galactose.

Subunit Composition of OLLafs and OLLafl Al- 
Table 2. Inhibition of OLLafs-, OLLafl- and Recombinant Lectins-Induced Hemagglutination by Saccharides ${ }^{a)}$

\begin{tabular}{|c|c|c|c|c|c|c|c|}
\hline \multirow{2}{*}{ Saccharide } & \multicolumn{7}{|c|}{ Concentration $^{b)}(\mathrm{mM})$} \\
\hline & afs & afl & rafl-H & rafl-L & mafl-L ${ }^{123}$ & mafl-L ${ }^{131}$ & mafl-L ${ }^{136}$ \\
\hline D-Galactose & 37.5 & 0.94 & 75 & 150 & 37.5 & $>150$ & $>150$ \\
\hline D-Glucose & 1.88 & 150 & 4.7 & 4.7 & 75 & $>150$ & 9.4 \\
\hline D-Mannose & 4.69 & $>150$ & NT & NT & NT & NT & NT \\
\hline L-Rhamnose & $>150$ & 0.01 & NT & NT & NT & NT & NT \\
\hline D-GalNAc & $>150$ & $>150$ & NT & NT & NT & NT & NT \\
\hline D-GlcNAc & 0.94 & $>150$ & 18.8 & 4.7 & 75 & $>150$ & 4.7 \\
\hline Lactose & 3.75 & 1.88 & 18.8 & 18.8 & 75 & $>150$ & 37.5 \\
\hline Sucrose & 37.5 & $>150$ & 37.5 & 18.8 & 37.5 & $>150$ & 9.4 \\
\hline Melibiose & 1.88 & 0.94 & 4.7 & 4.7 & 37.5 & $>150$ & 4.7 \\
\hline Lactulose & $>150$ & 0.94 & NT & NT & NT & NT & NT \\
\hline
\end{tabular}

a) Lectins abbreviated are; afs, OLLafs; afl, OLLafl; rafl-H and $-\mathrm{L}$, recombinant OLLafl-H and $-\mathrm{L}$, respectively; mafl- $\mathrm{L}^{123}$, $-\mathrm{L}^{131}$ and $-\mathrm{L}^{136}$, mutant lectins replaced Cys ${ }^{123}$ $\mathrm{Cys}^{131}$ and $\mathrm{Cys}^{136}$ to Ala, respectively. D-GalNAc, $N$-acetyl-D-galactosamine; D-GlcNAc, $N$-acetyl-D-glucosamine. NT, not tested. $b$ ) Minimum concentration of saccharide required for complete inhibition.

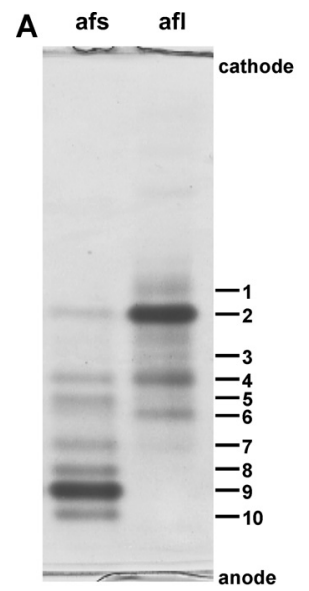

B 124
afl-H SYPSCPSrqWtKnGqR $\cdots$ CDnkrGFICSFKLC
afl-L SYPSCPSd-WiKsGeR $\cdots$ CDqeIGFICSFKLC

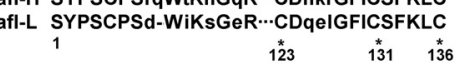

\section{C}

\begin{tabular}{|c|c|c|c|c|}
\hline Band No. & afs & afl & $R m$ & Subunit \\
\hline 1 & - & + & 0.46 & $\mathrm{H}$ and L \\
\hline 2 & + & + & 0.51 & $\mathrm{H}$ and $\mathrm{L}$ \\
\hline 3 & - & \pm & 0.58 & $\mathrm{~L}$ \\
\hline 4 & + & + & 0.63 & $\mathrm{~L}$ \\
\hline 5 & + & - & 0.68 & $\mathrm{~L}$ \\
\hline 6 & - & + & 0.70 & $\mathrm{H}$ and L \\
\hline 7 & + & \pm & 0.77 & $\mathrm{~L}$ \\
\hline 8 & + & - & 0.82 & $\mathrm{~L}$ \\
\hline 9 & + & - & 0.86 & $\mathrm{~L}$ \\
\hline 10 & + & - & 0.90 & $\mathrm{~L}$ \\
\hline
\end{tabular}

Fig. 3. Native-PAGE Pattern and Subunit Compositions of OLLs

Polyacrylamide gel electrophoresis was performed by using $7 \%$ gel at $\mathrm{pH} 9.5$ followed by Western blotting at $60 \mathrm{~V}$ for $3 \mathrm{~h}$ on polyvinylidene fluoride membrane with Coomassie Brilliant Blue staining (A). Digest sequences of H- and L- subunit of OLLafl were noted (B). Different amino acid residues between $\mathrm{H}$ - and L-subunit were cited with small letters. One gap was needed to obtain a sequence alignment. Abbreviated middle regions were indicated by dots. The results of N-terminal sequence analyses of each blotted band were summarized (C). +, obviously visible; \pm , faint stained; - , invisible; $R \mathrm{~m}$, relative mobility. " $\mathrm{H}$ and $\mathrm{L}$ " means the sequence result containing both of $\mathrm{H}$ - and L-subunit.

though either OLLafs or OLLafl was estimated to be homogeneous and to be a dimer on SDS-PAGE (Fig. 2B), each lectin showed multiple bands with distinct pattern on nativePAGE (Fig. 3A). As shown in Fig. 3B, the differences between amino acid sequences of $\mathrm{H}-$ and L-subunit of OLLafl were confined to two limited portions at $\mathrm{N}$ - and $\mathrm{C}$-terminal area. Therefore, whether the protein bands on native-PAGE contain H- and/or L-subunit can be revealed by amino acid sequence analysis of N-terminal 15 residues. Figure 3C shows the relative mobility $(R \mathrm{~m})$ and $\mathrm{N}$-terminal sequence ( $\mathrm{H}$ or $\mathrm{L}$ ) of blotted bands. Although OLLafs was remarkably different from OLLafl in native-PAGE pattern, three bands (No. 2, 4, 7) were involved in both lectins. Band No. 1, 2 and 6 was composed of $\mathrm{H}-$ and L-subunit, but remaining bands contained only L-subunit. Since each main band of OLLafs (No. 9) and OLLafl (No. 2) was composed of L-subunit and $\mathrm{H} / \mathrm{L}$-subunit, respectively, and since OLLafl included almost all bands composed of $\mathrm{H} / \mathrm{L}$-subunit, but OLLafs included bands composed of L-subunit excepting No. 2, it is sup-

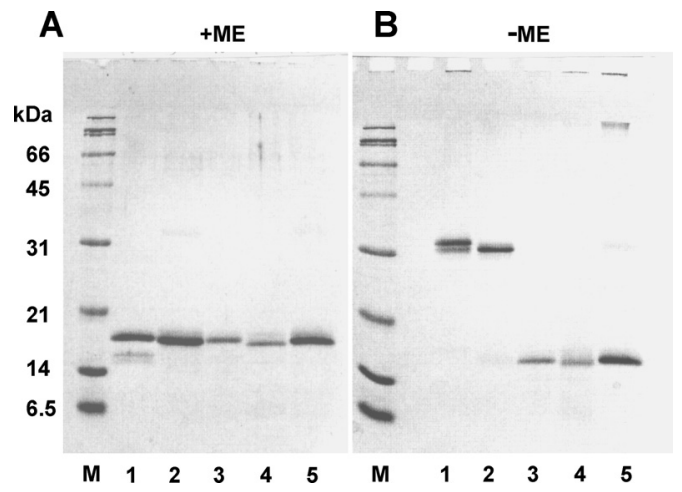

Fig. 4. SDS-PAGE Pattern of Recombinant OLLafl and Its Mutants

SDS-polyacrylamide gel electrophoreses were performed using homogeneous $15 \%$ gel with (A) or without (B) 2-mercaptoethanol (ME). Lane 1, rOLLafl-H; lane 2, rOLLafl-L; lane 3, mOLLafl-L ${ }^{123}$; lane 4 , mOLLafl-L ${ }^{131}$; lane 5, mOLLafl-L ${ }^{136}$. M, marker proteins.

ported that OLLafs is a homodimer of L-subunit and OLLafl is a heterodimer of $\mathrm{H} / \mathrm{L}$-subunit.

Preparation of Recombinant OLLs To explore the subunit interaction of OLLafl, we expressed the recombinant proteins, rOLLafl-H and -L, and the L-subunit mutants, which were replaced three Cys residues at $\mathrm{C}$-terminal region with Ala by site-directed mutagenesis (mOLLafl-L ${ }^{123}$, mOLLafl-L ${ }^{131}$ and mOLLafl- ${ }^{136}$ ). Although rOLLafl-L and its three mutants were obtained as soluble protein from inclusion bodies, rOLLafl-H became insoluble during refolding steps causing remarkable loss of yield (data not shown). These five recombinant proteins migrated at the positions of monomeric form on SDS-PAGE under reducing conditions (Fig. 4A). Under non-reducing conditions, rOLLafl-H and -L behaved as dimeric form, whereas three mutants migrated at monomeric form (Fig. 4B). Thus, mutant lectins were interfered to make an intermolecular disulfide bond. The extra band was observed in the preparations of rOLLafl-H (Figs. $4 \mathrm{~A}$ and $\mathrm{B}$, lane 1). Although the reason is still unclear, it is possible that rOLLafl-H was partially digested by endogenous proteases from host cells or that a misfolded rOLLafl-H was contaminated and migrated to a different position on SDS-PAGE.

Hemagglutinating Activity and Sugar Specificity of Recombinant OLLs As shown in Table 1, the hemagglutinating activities of intact lectins were lower than those of recom- 
binant or mutant lectins (Table 1). Namely, the minimum concentration of rOLLafl-L required for positive hemagglutination was 250 times lower than that of OLLafl. It is possible that the activities of OLLafs and OLLafl were reduced during purification steps. In another way, since no contaminant was observed in the preparations of OLLafs and OLLafl on SDSPAGE (Fig. 2), some kinds of low molecular weight inhibitors might be co-purified with the lectins and might diminish their activities. rOLLafl-H and -L showed hemagglutinating activity only existing as a dimeric form, because no activity was observed in monomeric protein obtained as a misfolding form (data not shown). However, three mutants were active even in a monomeric form (Fig. 4, Table 1). The activity mOLLafl$\mathrm{L}^{136}$ was comparable to rOLLafl-L, while mOLLafl-L ${ }^{123}$ and mOLLafl-L ${ }^{131}$ were lower than that of rOLLafl-H (Table 2). Therefore, Cys ${ }^{136}$ may not participate in the hemagglutinating activity of rOLLafl-L, but $\mathrm{Cys}^{123}$ and $\mathrm{Cys}^{131}$ may partially contribute this activity. All recombinant lectins reduced their hemagglutinating activities by adding $5 \mathrm{~mm}$ EDTA, so that they are $\mathrm{Ca}^{2+}$-dependent lectins. The reducing rate of $\mathrm{mOLL}$ afl- $\mathrm{L}^{131}$ was lower than the other lectins. In contrast, OLLafl showed $\mathrm{Ca}^{2+}$-independent activity (Table 1 ). The reason of this phenomenon is still unclear so that the properties of recombinant $\mathrm{H} / \mathrm{L}$-dimer will need to understand it.

Although hemagglutination inhibition profiles of rOLLaflL, rOLLafl-H and mOLLafl-L ${ }^{136}$ were similar, mOLLafl$\mathrm{L}^{131}$-induced hemagglutination was not inhibited by any sugars tested even at concentrations of $150 \mathrm{~mm}$. Therefore, $\mathrm{Cys}^{131}$ may directly contribute to the sugar-binding capacity of OLLafl. Affinities of mOLLafl-L ${ }^{123}$ for these sugars were lower than the others (Table 2). In addition, minimum concentrations of sugars required for complete inhibition of recombinant lectin-induced hemagglutination were slightly higher than those for intact lectins.

\section{DISCUSSION}

In the previous study, we obtained the heterodimeric lectin from $O$. lanceolatus eggs (OLLafl), which was eluted from asialofetuin-Sepharose column with lactose (unpublished data). OLLafl was composed of very similar two subunits $(\mathrm{H}$ and $\mathrm{L}, 95 \%$ identical) according to the cDNA sequence analysis. CTLD, a characteristic module of C-type lectins, and the positions of Cys residues were well conserved in both subunits. Therefore, OLLafl was considered to be a C-type galactose-binding lectin. When sucrose solution was used to remove contaminants from the asialofetuinSepharose column, OLLafs (not OLLafl) was contained in effluent from the column (Fig. 1C). OLLafs showed slightly different SDS-PAGE pattern on a homogeneous $20 \%$ gel compared with OLLafl (Fig. 2A). Since N-terminal sequence analyses of bands from OLLafs separated by using homogeneous gel indicated that it did not contain H-subunit, it was estimated to be a homodimer of L-subunit.

In addition, native-PAGE (Fig. 3A) and 2-dimentional electrophoresis (data not shown) revealed that OLLafs and OLLafl contained multiple components ( $c a$. 10 bands) consisting of $\mathrm{H}$ - or L-subunit with various $\mathrm{pI}$. Although " $\mathrm{H}$ and L" band (No. 2) was observed in OLLafs, its content was very low. It is suggested that OLLafs is almost composed by L-subunit. On the other hand, OLLafl contained both $\mathrm{H}$ - and
L-subunit equally (Fig. 3C) corresponding to the result of SDS-PAGE (Fig. 2A). Although these dimeric structures might be formed by single disulfide bond, it is difficult to understand why such molecular heterogeneity was observed in these lectins. The majority of the group VII C-type lectins, comprising free CTLD, reported so far are snake venoms e.g. Habu IX/X-binding protein. ${ }^{20)}$ They have various effects on either blood coagulation or platelet aggregation. ${ }^{21)}$ Among these, purpreotin isolated from Trimeresurus purpureomaculatus venom has a dimeric structure of $\alpha, \beta$-heterodimer with disulfide bond. In addition, $\alpha$ and $\beta$ chains of purpreotin are held together with electrostatic interactions and with hydrophobic interactions between dimeric molecules, respectively. It is so-called di-dimer structure, and in addition, there are some venom lectins known to form tri-dimer ${ }^{22}$ and tetradimer $^{23)}$ structure. They have been controversial about their diversity of structures and functions together with dimerization by $3 \mathrm{D}$ domain swapping phenomenon. ${ }^{24)}$ It is possible that $\mathrm{H}$ - and L-subunit randomly associate each other by hydrophilic or hydrophobic interaction excepting disulfide bond and may form multiple lectin molecules possessing various pI values.

Although hemagglutinating activities were not much different between OLLafs and OLLafl, the addition of EDTA caused diminishing the activity of OLLafs. In contrast, no change was observed in OLLafl (Table 1). Tasumi et al. have reported that hemagglutinating activity of AJL-2 was not influenced by the addition of EDTA. ${ }^{5}$ Generally, C-type lectin has two $\mathrm{Ca}^{2+}$ binding sites. Typically in mannose-binding lectin (MBP), the first and second sites are formed by amino acid residues of $\mathrm{Asp}^{161} \mathrm{Glu}^{165}, \mathrm{Asp}^{188}$ and $\mathrm{Asp}^{194}$, and by those of $\mathrm{Glu}^{185}$, Asn ${ }^{187}, \mathrm{Glu}^{193}, \mathrm{Asn}^{205}$ and $\mathrm{Asp}^{206}$, respectively. The latter also takes part in sugar-binding. ${ }^{25)}$ In AJL-2, amino acid residues corresponding to $\mathrm{Asp}^{161}$ and $\mathrm{Glu}^{165}$ of MBP are replaced with Ala and Gly, respectively, which are missed out the carboxyl group of side chain, so that it behaves as $\mathrm{Ca}^{2+}$-independent lectin. In OLLafl, amino acid residues corresponding to Glu ${ }^{165}, \mathrm{Asp}^{188}$ and $\mathrm{Asp}^{194}$ of MBP were also replaced with Asp, Asn and Pro, indicating that two carboxyl groups were lost as well as AJL-2. Thus, OLLafl possessed hemagglutinating activity in the presence of EDTA. However, EDTA diminished the activity of OLLafs. As shown in Fig. 3B, OLLafl-H had preferentially basic amino acid residues in the $\mathrm{N}$ - and $\mathrm{C}$-terminal diverse regions, but the corresponding regions of OLLafl-L were filled in acidic amino acid residues. Thus, an electrostatic and repulsive interaction may occur in OLLafl $(\mathrm{H} / \mathrm{L})$ and OLLafs $(\mathrm{L} / \mathrm{L})$, respectively. Consequently, the subunit conformation may be diversely changed.

OLLafs showed clearly different sugar-binding profiles compared with OLLafl (Table 2). Thus, these lectins have distinct binding property for erythrocytes. It is interesting that two lectins having similar structure with different property were eluted from asialofetuin-Sepharose by switching hapten sugar.

To clarify the interaction between structure and lectin activity of OLLafl was performed using recombinant OLLafl (rOLLafl-H and -L), and mutants (mOLLafl-L ${ }^{123}$, mOLLafl$\mathrm{L}^{131}$ and mOLLafl-L $\left.{ }^{136}\right)$. As shown in Fig. 4, all mutants failed to form intermolecular disulfide bond, and showed monomeric form on SDS-PAGE under non-reducing condi- 
tions. If more than dimeric form is needed for hemagglutinating activity, it may be supplied by multimerization system with non-covalent bond as described above.

Although rOLLafl-H and -L showed no significant difference in sugar specificities, mOLLafl-L ${ }^{123}$ and mOLLafl-L ${ }^{131}$ were considerably reduced sugar-binding activity (Table 2). Therefore, each $\mathrm{Cys}^{123}$ and $\mathrm{Cys}^{131}$ may form CTLD loops and may participate in sugar-binding reactions. In contrast, mOLLafl-L ${ }^{136}$ had sugar-binding activity, indicating that Cys $^{136}$ may not contribute to sugar-binding but may take part in intermolecular disulfide bond. Interestingly, mOLLafl-L ${ }^{136}$ lost only galactose-binding activity. This result suggests that dimeric structure via disulfide bond may play an important role in binding between OLLafl and galactose.

We presumed that a single intermolecular disulfide bond is formed between C-terminal ends of both subunits based on the architecture of the other C-type lectins. However, from the results of Fig. 4, dimeric form was not observed in any mutants. Probably, misfolding may be occurred by replacement of Cys with Ala during the refolding steps. Different loops were consequently formed between the most convenient residues for three intramolecular disulfide bonds, and residual one Cys (probably excepting $\mathrm{Cys}^{136}$ ) could not form disulfide bond for some reasons.

Although many L-rhamnose-binding lectins (RBLs) have been found in fish eggs, only a few C-type lectins were found so far. For example, C-type lectin was not found in catfish eggs. It is interesting that $O$. lanceolatus (shishamo smelt) eggs contain both lectins, and this inevitability will need to be solved understanding the significance of fish egg lectins.

\section{REFERENCES}

1) Dodd R. B., Drickamer K., Glycobiology, 11, 71R_79R (2001).
2) Iobst S. T., Wormald M. R., Weis W. I., Dwek R. A., Drickamer K., J. Biol. Chem., 269, 15505-15511 (1994).

3) Dong C.-H., Yang S.-T., Yang Z.-A., Zhang L., Gui J.-F., Dev. Biol., 265, 341-354 (2004).

4) Richards R. C., Hudson D. M., Thibault P., Ewart K. V., Biochim. Biophys. Acta, 1621, 110-115 (2003).

5) Tasumi S., Ohira T., Kawazoe I., Suetake H., Suzuki Y., Aida K., J. Biol. Chem., 277, 27305-27311 (2002).

6) Savan R., Endo M., Sakai M., Mol. Immunol., 41, 891-899 (2004).

7) Zarkadis I. K., Nikolakopoulou K. Direct submission data: AF363271. 1.

8) Zhang H., Robison B., Thorgaard G. H., Ristow S. S., Biochim. Biophys. Acta, 1494, 14-22 (2000).

9) Fujiki K., Bayne C. J., Shin D. H., Nakao M., Yano T., Fish Shellfish Immunol., 11, 275-279 (2001).

10) Mistry A. C., Honda S., Hirose S., Biochem. J., 360, 107-115 (2001).

11) Vitved L., Holmskov U., Koch C., Teisner B., Hansen S., Skjødt K., Immunogenetics, 51, 955-964 (2000).

12) Spiro R. G., J. Biol. Chem., 237, 646-652 (1962).

13) March S. C., Parikh I., Cuatrecasas P., Anal. Biochem., 60, 149-152 (1974).

14) Hosono M., Matsuda K., Kawauchi H., Takayanagi Y., Shiokawa H., Mineki R., Murayama K., Nitta K., Biomed. Res., 13, 443-449 (1992).

15) Laemmli U. K., Nature (London), 227, 680 -685 (1970).

16) Davis B. J., Ann. N.Y. Acad. Sci., 121, $404-427$ (1964).

17) Lowry O. H., Rosenbrough N. J., Farr A. L. Randall R. J., J. Biol. Chem., 193, 265-275 (1951).

18) Nitta K., Takayanagi G., Kawauchi H., Chem. Pharm. Bull., 31, 315320 (1983).

19) Ewart K.V., Li Z., Yang D. S., Fletcher G. L., Hew C. L., Biochemistry, 37, 4080-4085 (1998).

20) Atoda H., Morita T., J. Biochem. (Tokyo), 106, 808 -813 (1989).

21) Li X., Zheng L., Kong C., Kolatkar P. R., Chung M. C. M., Arch. Biochem. Biophys., 424, 53-62 (2004).

22) Leduc M., Bon C., Biochem. J., 333, 389-393 (1998).

23) Fukuda K., Mizuno H., Atoda H., Morita T., Biochemistry, 39, 19151923 (2000).

24) Mizuno H., Fujimoto Z., Koizumi M., Kano H., Atoda H., Morita T., Nature Struct. Biol., 4, 438- 441 (1997).

25) Day A. J., Biochem. Soc. Trans., 22, 83-87 (1994). 